Open Access

\title{
Characterization and applications of Nanobodies against human procalcitonin selected from a novel naïve Nanobody phage display library
}

\author{
Junrong Yan', Pingyan Wang ${ }^{1}$, Min Zhu' ${ }^{1}$ Guanghui Li', Ema Romão ${ }^{4}$, Sheng Xiong ${ }^{3^{*}}$ and Yakun Wan ${ }^{1,2^{*}}$
}

\begin{abstract}
Background: Nanobodies (Nbs) are single-domain antigen-binding fragments derived from the camelids heavy-chain only antibodies (HCAbs). Their unique advantageous properties make Nbs highly attractive in various applications. The general approach to obtain Nbs is to isolate them from immune libraries by phage display technology. However, it is unfeasible when the antigens are toxic, lethal, transmissible or of low immunogenicity. Naïve libraries could be an alternative way to solve the above problems.

Results: We constructed a large camel naïve phage display Nanobody ( $\mathrm{Nb}$ ) library with great diversity. The generated library contains to $6.86 \times 10^{11}$ clones and to our best of knowledge, this is the biggest naive phage display Nb library. Then Nbs against human procalcitonin (PCT) were isolated from this library. These Nbs showed comparable affinity and antigen-binding thermostability at $37^{\circ} \mathrm{C}$ and $60^{\circ} \mathrm{C}$ compared to the PCT Nbs from an immune phage-displayed library. Furthermore, two PCT Nbs that recognize unique epitopes on PCT have been successfully applied to develop a sandwich enzyme-linked immunosorbent assay (ELISA) to detect PCT, which showed a linear working range from 10-1000 $\mathrm{ng} / \mathrm{mL}$ of PCT.
\end{abstract}

Conclusion: We have constructed a large and diverse naïve phage display Nb library, which potentially functioning as a good resource for selecting antigen-binders with high quality. Moreover, functional Nbs against PCT were successfully characterized and applied, providing great values on medical application.

Keywords: Bactrian camel, VHH, Naïve phage-displayed library, Human procalcitonin, Biotin-Streptavidin-System, Sandwich ELISA

\section{Background}

Since their discovery, antibodies have become excellent candidates for research, diagnostic and therapeutic applications due to their high affinity and specificity characteristics [1,2]. Most applications rely on conventional immunoglobulin G (IgG) molecules. These antibodies, produced by $\mathrm{B}$ lymphocytes, comprise two identical

\footnotetext{
* Correspondence: xiongsheng@jnu.edu.cn; ywansystemsbiology@gmail.com ${ }^{3}$ Institute of Biomedicine \& National Engineering Research Center of Genetic Medicine, College of Life Science and Technology, Jinan University, Guangzhou 510630, PR China

${ }^{1}$ The Key Laboratory of Developmental Genes and Human Disease, Ministry of Education, Institute of Life Sciences, Southeast University, Nanjing 210096, PR China

Full list of author information is available at the end of the article
}

heavy chains and two identical light chains [3]. The molecular weight of an IgG is approximately $160 \mathrm{kDa}$, and it is a complex molecule consisting of heavy and light chains, which together form the antigen binding site [4]. However, some limitations of the IgG, such as its large size and complicated structure, the time consuming and costly production, unstable behaviors and their reliance on mammalian cells expression, [5] create numerous problems on practical applications. An alternative approach is to use some antibody fragments, such as antigen-binding fragments (Fabs) and single-chain variable fragments (scFvs). 
With the rapid development of molecular gene engineering techniques, Fabs $(50 \mathrm{kDa})$ and scFvs $(27 \mathrm{kDa})$ have been widely used as alternatives to classic antibodies. These antibody fragments can maintain the specificity to antigens. Fabs are the oldest class of monoclonal antibody derived fragments, which are clearly thoroughly explored [6]. Unfortunately, due to their relatively large size of approximately $50 \mathrm{kDa}$, Fabs yield large oligomeric molecules [7]. scFvs are the smallest intact antigen-binding fragments that can be derived from a conventional IgG molecule [4]. They are recombinant antigen-binding fragments in which the variable regions of light and heavy chains are combined into a single polypeptide. The small size of scFvs permits penetration into obstructed locations that inaccessible for full-size conventional antibodies, such as tumors [8]. However, scFvs tend to form bivalent or higher oligomers [7]. Additionally, the production of these fragments is difficult due to their low yield, poor solubility and stability [9]. Moreover, their susceptibility to temperature and $\mathrm{pH}$ [10] make them unsuitable for use in biosensors as diagnostics or as therapeutic candidates.

Aside from classic IgGs, researchers found that certain animals, such as camelids and sharks, [11,12] are able to produce a unique kind of antibody naturally lacking light chains, termed heavy chain-only antibodies (HCAbs). The antigen-binding sites of the HCAbs are composed of two single variable domains, named the variable domain of heavy chain of the heavy chain-only antibody ( $\mathrm{VHH}$ ) [4]. VHHs are distinguished from the conventional IgG and various IgG derivatives by their unique properties of molecular size (2.5 nm in diameter and $4 \mathrm{~nm}$ in height), [13] therefore, it is also known as Nanobody $(\mathrm{Nb})$. Nanobodies (Nbs) are the smallest fully functional antigenbinding fragments [13]. Due to their small size (15 kDa) and single-domain nature, $\mathrm{Nbs}$ are ideal entities for basic research, biosensor and therapeutic applications. This monomeric antigen-binding fragment shows several special features in terms of their high thermal and conformational stability, [14] high aqueous solubility, straightforward production in micro-organisms, [9] resistance to acid and alkaline $\mathrm{pH},[15]$ excellent affinity and specificity, [13] ease of manufacture [7] and low immunogenicity. In addition, Nbs exhibit superior body distribution, tissue penetration and faster blood clearance rate [9]. These advantageous properties make Nbs attractive and valuable tools for various applications.

Generally, Nbs can be isolated from an immune $\mathrm{Nb}$ library. VHHs genes can be reverse-transcribed from the mRNA of peripheral blood lymphocytes (PBLs) of animals that were immunized with the antigens of interest. Phage display technology is the most popular method to isolate antigen-binding clones [16]. Although camels, llamas and dromedaries have all been immunized to produce antigen binders in many studies, [17-19] this routine way is often unrealistic when the antigens are toxic, lethal, transmissible, of low immunogenicity, or even nonimmunogenic small molecular compounds $[16,20]$. In addition, the immunization process is timeconsuming and normally takes months to raise a satisfactorily immune response [16]. Alternatively, naïve phage display libraries could solve the above problems. Nonimmunized camels can be used to generate unbiased or non-immune libraries, from which antigen binders of interest can be selected.

In this study, based on the VHHs genes, which were amplified from the mRNA of peripheral blood lymphocytes (PBLs) of twenty four non-immunized bactrian camels and a spleen from another non-immunized bactrian camel, we successfully constructed a large naïve phage-displayed library with great diversity. The library contains $6.86 \times 10^{11}$ clones, with an insertion rate of clones having a $\mathrm{VHH}$ of nearly $100 \%$. Then Nbs against human procalcitonin (PCT) were isolated from this phage-displayed library. PCT is a highly valuable diagnostic biomarker of bacterial infections, which is frequently used as a reliable marker to support the risk evaluation of seriously sick people progressing to severe sepsis and septic shock [21,22]. Furthermore, by comparing the PCT Nbs from an immune phage-displayed library constructed in our previous study, [23] the naïve library derived PCT Nbs showed comparable affinity and antigen-binding thermostability at $37^{\circ} \mathrm{C}$ and $60^{\circ} \mathrm{C}$. In addition, two Nbs recognizing unique epitopes of PCT, were used to develop a sandwich enzyme-linked immunosorbent assay (ELISA) to detect the bacterial protein. Consequently, the Nbs from the naive library were successfully used for capture and detection of native PCT in the ELISA based diagnostic assay allowing the sensitive measurement of the disease marker. Altogether, such a large and diverse naïve phage-displayed library represents a good resource for selecting functional binders with high affinity and specificity, hence being an essential alternative to immune libraries.

\section{Results and discussion}

\section{Construction of a naïve VHH library}

The general way to obtain Nbs is screening the antigenspecific Nbs from an immune library. However, when the antigens are toxic, low immunogenic, or nonimmunogenic small molecular compounds, immunization will not be practical. Such circumstances also impose great challenges for conventional antibody generation. Additionally, the process takes more than one month for camels to raise a sufficiently high immune response. Isolating Nbs from a naïve library could be an alternative way. To construct a large and highly diverse naïve $\mathrm{Nb}$ library, as shown in Figure 1, total RNA was isolated from PBLs collected from twenty four non- 


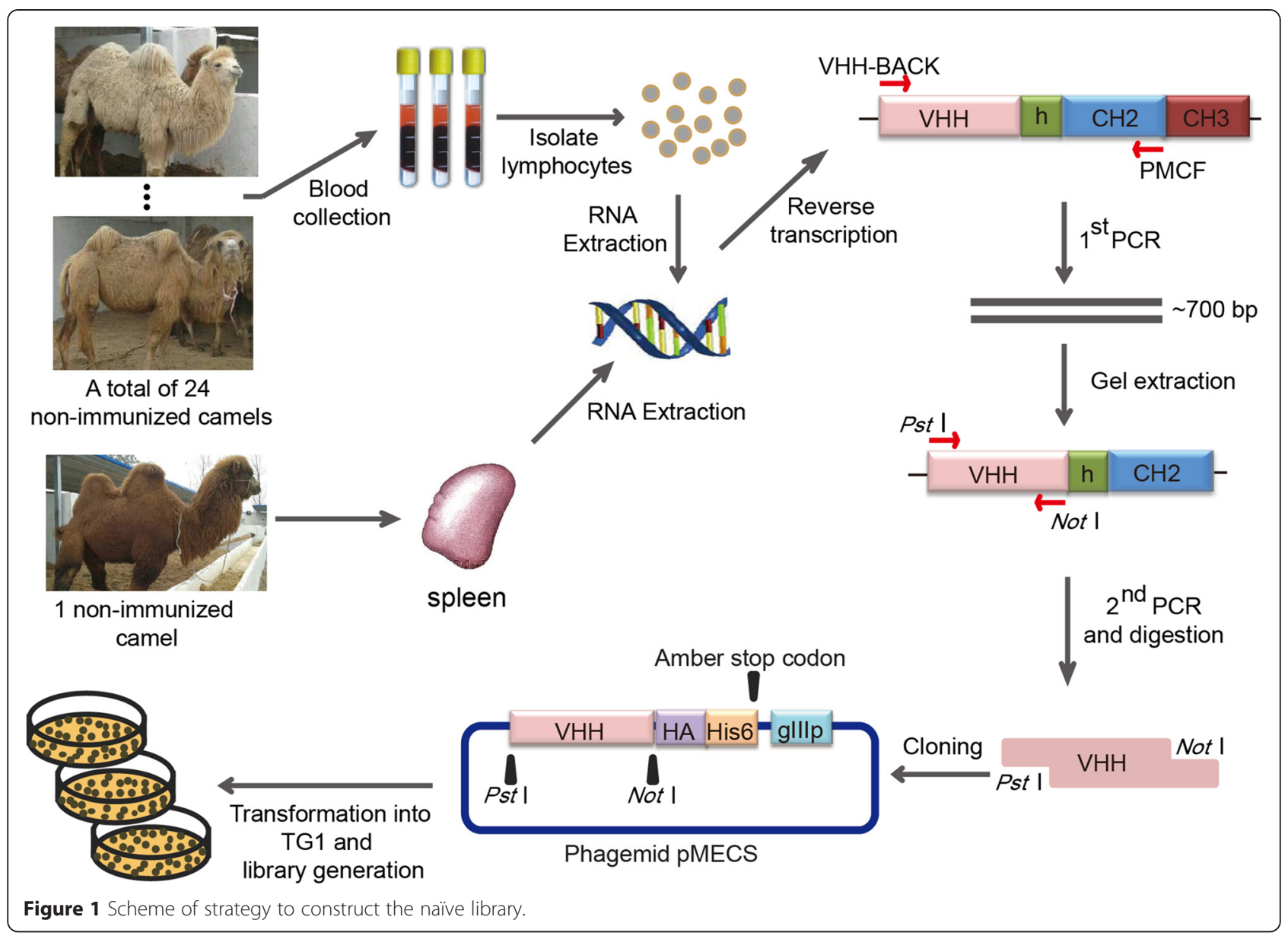

immunized bactrian camels and a spleen from another non-immunized camel. Amplicons spanning the VHH$\mathrm{CH} 2$ exons with a size of $700 \mathrm{bp}$ were generated during the primary PCR (Figure 2A). The VHH encoding gene fragments were then amplified by the second PCR (Figure 2B) with the $700 \mathrm{bp}$ fragment purified with gel extraction as the template using degenerated primers that introduced the Pst I and Not I restriction sites (Figure 1). The Pst I and Not I double-digested amplicons were cloned into the phagemid vector pMECS allowing the expression of C-terminal Hemagglutinin (HA)-His6-tagged Nbs, after that, the recombinant vector was electro-transformed into competent TG1 cells. Dilution plating of the cultured library indicated a total size of $6.86 \times 10^{11}$ colony-forming units (CFU) (Figure 2C), being the largest phage-displayed $\mathrm{Nb}$ library to our understanding. PCR analysis of 24 randomly picked clones demonstrated a frequency of clones having a complete VHH insert of $100 \%$ (Figure 2D), which meant the functional capacity of the library was very high. The sequencing results of the 300 randomly picked clones showed the library has a high diversity, 9 groups containing 20 clones exhibited the same sequence in CDR3 regions with each other within the groups, and among them, 2 groups containing 4 clones showed the exactly same amino acids all through the whole $\mathrm{VHH}$ with each other within the groups. Thus, there are 289 kinds of different amino acids sequences in CDR3 regions among these 300 clones (Additional file 1: Figure S1-S10), which indicated the diversity was high. Overall, we have successfully constructed a naïve phage display $\mathrm{Nb}$ library of high quality for the following selection of Nbs against PCT.

\section{Selection of PCT Nbs}

By taking advantage of the high diversity of the naïve $\mathrm{Nb}$ library, we identified PCT Nbs by bio-panning to validate the quality of the library using phage display technology. As it is a very large library, we only took a small sample of the library to investigate whether good quality of binders can be isolated as a previous study did [24]. Although we did not screen the whole naïve library herein and it may lose the diversity of Nbs during selection, we were still able to retrieve PCT Nbs with comparable characteristics to those of the immune PCT Nbs according to the following results in this study, which in turn shows that the naïve library can be indeed a powerful and resourceful 
A
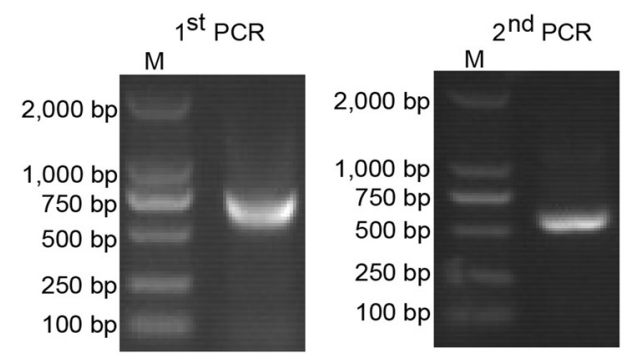

B
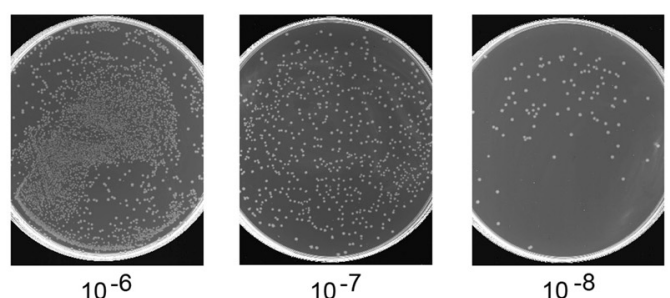

c

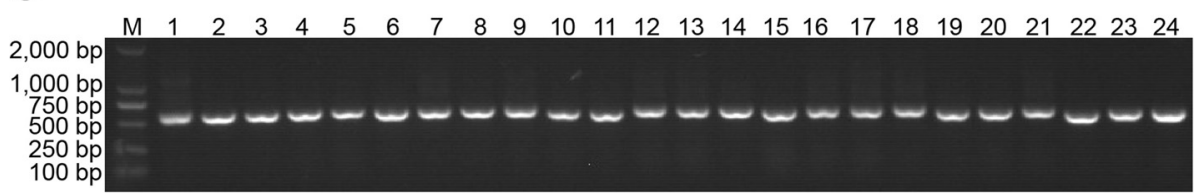

Figure 2 Construction of the naive library. (A)The VHH genes were obtained by two steps PCR. (B) The library size was measured by counting the colonies number after serial dilution. (C) 24 colonies were randomly picked to estimate the correct insertion rate of VHH genes by PCR amplification.

alternative to the immune libraries. During the screening, we calculated the relative enriching efficiency of phage particles eluted from wells coated with PCT versus those without antigens. Next, 95 individual colonies were randomly picked to identify specific Nbs by performing periplasmic extraction ELISA (PE-ELISA) after the $4^{\text {th }}$ round of panning. A total of 34 positive colonies with a binding ratio of more than 3 were identified. The positive colonies were sent for sequencing, and seven different sequences were obtained. Finally, the PCT-specific Nbs were classified into three families based on the diversity of their amino acid sequences in complementarity determining region (CDR) 3 (Figure 3A). These Nbs were named PCT $\mathrm{Nb} 1, \mathrm{Nb} 2$ and $\mathrm{Nb} 3$. In our previous study, we have obtained several anti-PCT Nbs from an immune library, which were successfully used to develop a Nb-based electrochemiluminescent immunosensor for sensitive detection of human PCT [23]. The amino acids sequences of CDR3 comparison between the two different libraries-derived PCT Nbs demonstrates that they do not share any sequence similarity. Thus far, we have successfully isolated diverse Nbs, indicating that the naive library is a great resource for Nbs selection. Moreover, we need to further assess the quality of the non-immune library by screening more antigens.

\section{Expression and purification of soluble Nbs}

In order to express the isolated Nbs, we transformed the recombinant phagemid from $E$. coli TG1 to WK6 cells.
TG1 cells suppress the amber stop codon between VHH and the gene III in phagemid pMECS, resulting in the expression of VHH-gene III fusions. However, WK6 is a non-suppressor strain and will not express the gene III fusion, but only the $\mathrm{VHH}$, which contains at its Cterminal end a HA-His6-tag. (Figure 3B). Soluble Nbs were extracted from the periplasm by osmotic shock and they were purified on a NI-NTA Superflow Sepharose column using $500 \mathrm{mM}$ imidazole for elution. SDS-PAGE analysis demonstrated the good quality of $\mathrm{Nbs}$ obtained with high purity (Figure $3 \mathrm{~B}$ ) and milligram quantities of production were yielded were yielded from $1 \mathrm{~L}$ of culture (Table 1).

\section{Binding thermostability analysis}

To further explore whether the naïve library-derived PCT Nbs were as good as the immune library-derived $\mathrm{Nbs}$ in thermostability, we performed binding thermostability analysis by ELISA with three PCT specific Nbs derived from the naive library and four target-specific Nbs isolated from the immune library. From the analysis, we found that Nb87 shows the lowest degree of thermal stability, since it could not maintain antigen binding when incubated for $1 \mathrm{~h}$ at $60^{\circ}$ $\mathrm{C}$ or $90^{\circ} \mathrm{C}$ (Figure $4 \mathrm{~A}, \mathrm{~B}, \mathrm{C}$ ). Among the naïve libraryderived PCT Nbs, Nb2 has a worse binding thermostability, but $\mathrm{Nb} 1$ and $\mathrm{Nb} 3$ could keep binding thermostability profile with three other immune library derived $\mathrm{Nbs}$ under the treatment at $37^{\circ} \mathrm{C}$ and $60^{\circ} \mathrm{C}$ (Figure $4 \mathrm{~A}, \mathrm{~B}$ ). However, when 


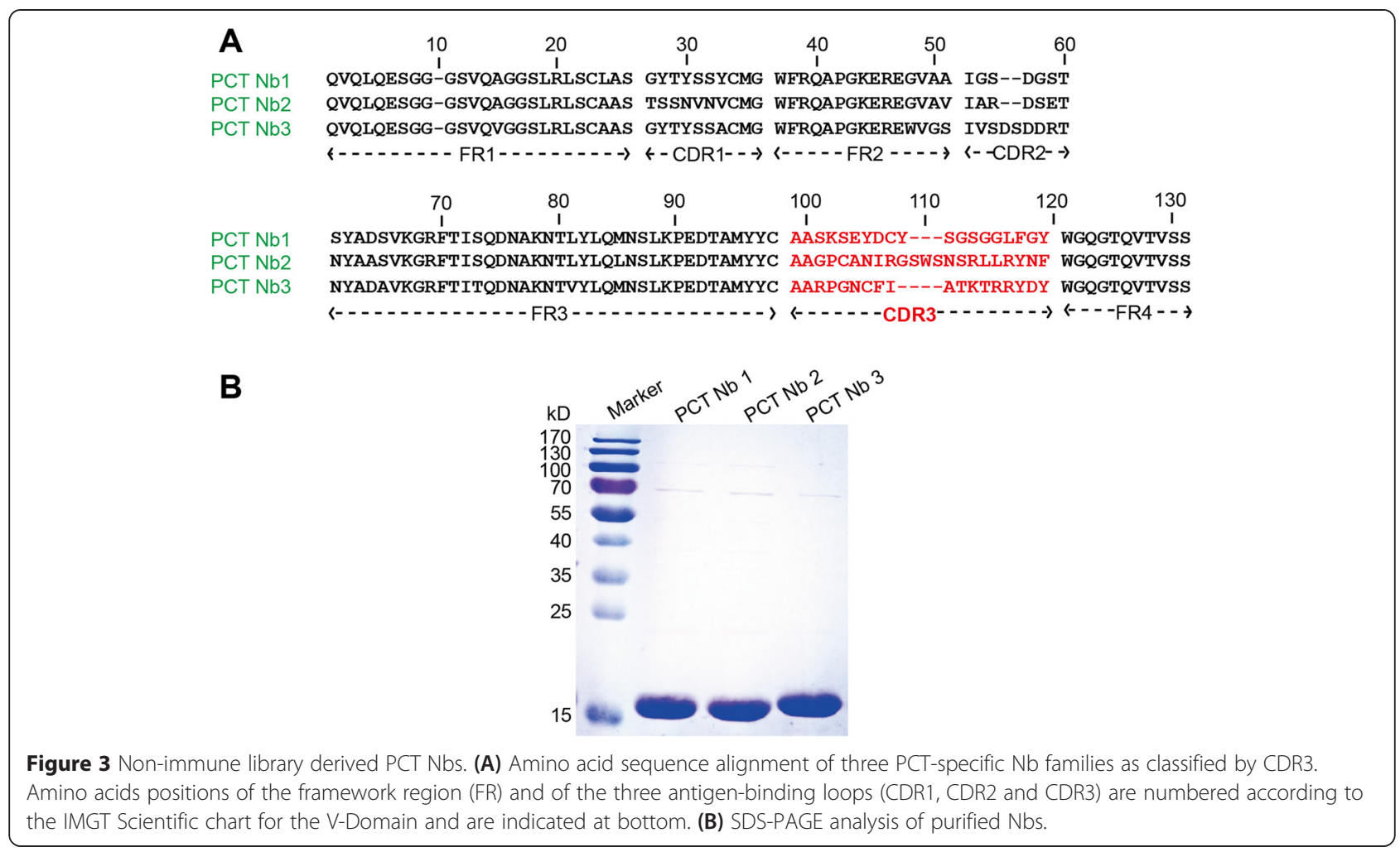

the Nbs were treated at $90^{\circ} \mathrm{C}$, the immune library-derived $\mathrm{Nb} 52, \mathrm{Nb} 85$ and Nb88 show better binding stability than the three $\mathrm{Nbs}$ from the naïve library (Figure 4C). In general, the binding thermostability of Nbs is individually different. According to the analysis, the naïve library-derived PCT Nbs could retain similar binding thermostability as the immune library derived $\mathrm{Nbs}$, at temperatures that were not particularly high. However, when incubated at $90^{\circ} \mathrm{C}$, we did not find one $\mathrm{Nb}$ from the naive library that exhibited similar antigen-binding thermostability as the immune library- derived Nbs. It is probably due to the lack of in vivo maturation when camels are immunized that the $\mathrm{Nbs}$ isolated from the non-immune libraries have a somewhat lower thermal stability. Nevertheless, it should be good enough for most applications, since temperatures above $60^{\circ} \mathrm{C}$ will not be experienced and also not for prolonged periods.

\section{Affinity analysis}

As we wanted to further prove the quality of the three $\mathrm{Nbs}$ selected from the naïve library, we measured their

Table 1 Properties of PCT Nbs from the naïve library and the immune library [23]

\begin{tabular}{|c|c|c|c|c|c|c|c|}
\hline VHH & $\mathrm{M}_{\mathrm{W}}^{\mathrm{a}}(\mathrm{kDa})$ & $\mathrm{pl}^{\mathrm{b}}$ & $\varepsilon^{c}(g / L)$ & Yield (mg/L) & $K_{o n}\left(M^{-1} s^{-1}\right)$ & $\mathrm{K}_{\mathrm{Off}}\left(\mathrm{s}^{-1}\right)$ & $K_{D}(M)$ \\
\hline PCT Nb1 & 15.47 & 6.03 & 1.964 & 7.5 & $4.16 \times 10^{4}$ & $5.10 \times 10^{-4}$ & $1.23 \times 10^{-8}$ \\
\hline PCT Nb2 & 15.18 & 8.73 & 1.833 & 4.5 & $1.47 \times 10^{5}$ & $1.97 \times 10^{-4}$ & $1.34 \times 10^{-9}$ \\
\hline PCT Nb3 & 16.08 & 7.19 & 1.954 & 6.0 & $4.31 \times 10^{4}$ & $3.83 \times 10^{-4}$ & $8.89 \times 10^{-9}$ \\
\hline PCT Nb20 & 16.05 & 6.13 & 2.498 & 7.1 & $2.85 \times 10^{4}$ & $9.52 \times 10^{-4}$ & $3.34 \times 10^{-8}$ \\
\hline PCT Nb45 & 15.71 & 7.95 & 2.027 & 4.9 & $1.64 \times 10^{3}$ & $4.02 \times 10^{-5}$ & $2.45 \times 10^{-8}$ \\
\hline PCT Nb50 & 16.12 & 7.17 & 2.577 & 8.7 & $2.39 \times 10^{4}$ & $6.34 \times 10^{-4}$ & $2.65 \times 10^{-9}$ \\
\hline PCT Nb52 & 15.59 & 6.70 & 2.367 & 9.3 & $2.32 \times 10^{4}$ & $6.10 \times 10^{-4}$ & $2.63 \times 10^{-8}$ \\
\hline PCT Nb85 & 16.07 & 7.17 & 2.578 & 6.4 & $2.11 \times 10^{4}$ & $8.21 \times 10^{-4}$ & $3.89 \times 10^{-9}$ \\
\hline PCT Nb86 & 16.18 & 8.36 & 2.317 & 5.5 & $2.52 \times 10^{4}$ & $8.65 \times 10^{-4}$ & $3.43 \times 10^{-9}$ \\
\hline PCT Nb87 & 16.64 & 6.38 & 1.825 & 10.3 & $4.18 \times 10^{5}$ & $2.58 \times 10^{-4}$ & $6.17 \times 10^{-9}$ \\
\hline PCT Nb88 & 15.63 & 8.80 & 1.925 & 7.6 & $1.99 \times 10^{4}$ & $8.05 \times 10^{-4}$ & $4.04 \times 10^{-9}$ \\
\hline
\end{tabular}

${ }^{\mathrm{a}}$ Molecular weight (Mw) includes $\mathrm{HA}$ and $\mathrm{His}_{6}$ tag.

$\mathrm{a}, \mathrm{b}, \mathrm{c}$ Theoretical isoelectric point $(\mathrm{pl}), \mathrm{Mw}$ and extinction coefficient $(\varepsilon)$ were calculated by the ExPAsy ProtParam Tool. 

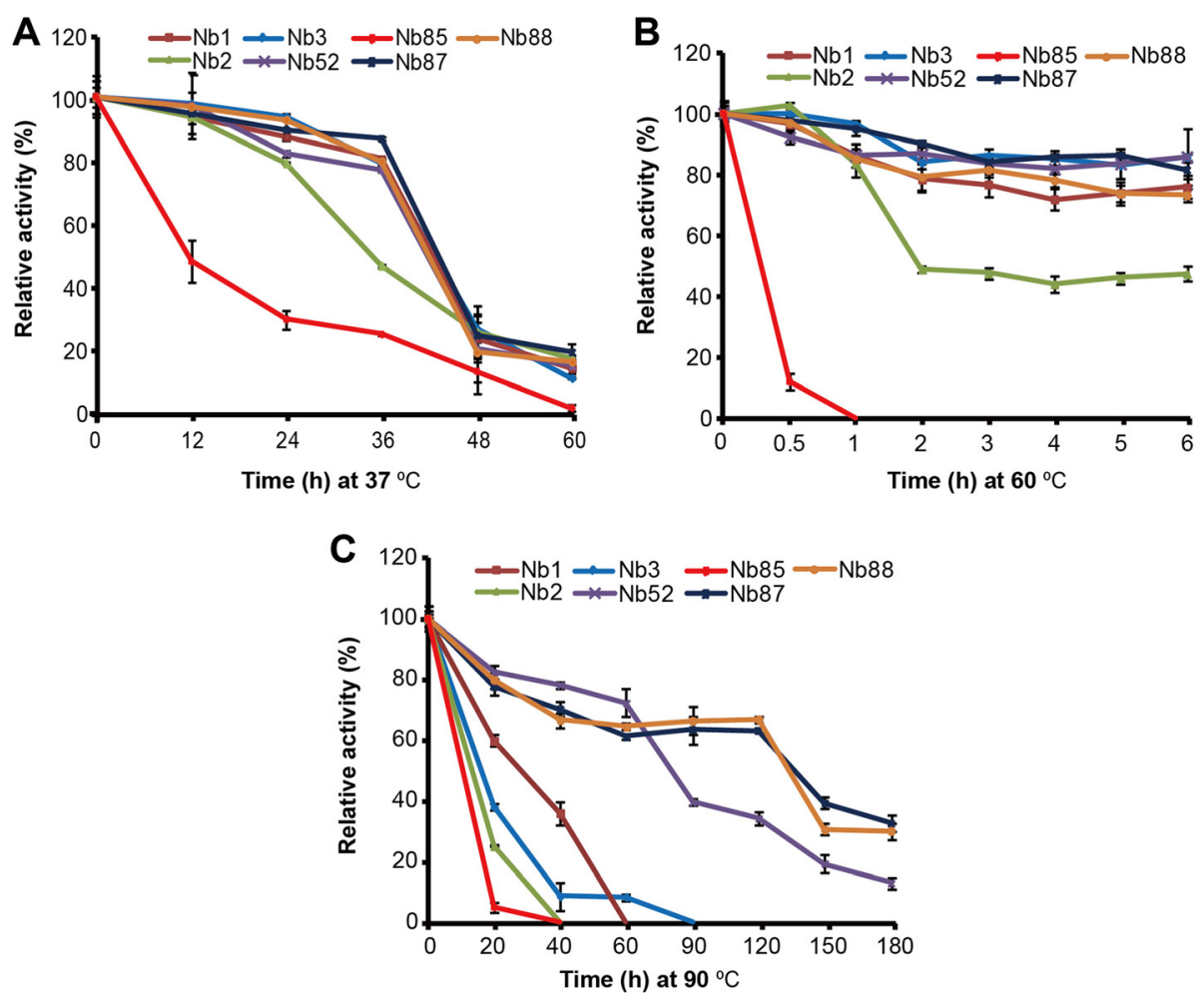

Figure 4 Binding thermostability analysis. The PCT Nbs from the naïve and the immune libraries were incubated at (A) $37^{\circ} \mathrm{C}$, (B) $60^{\circ} \mathrm{C}$ or $(\mathbf{C}) 90^{\circ} \mathrm{C}$ for different times to analyze the binding thermostability by ELISA. The activity of the Nbs never been treated was regarded as $100 \%$ and three independent experiments were performed.

affinities by SPRi binding analysis using PlexArray ${ }^{\circ} \mathrm{HT}$ system. As the sensorgrams (Figure 5A, B and C) show, these three Nbs presented their equilibrium dissociation constants $\left(\mathrm{K}_{\mathrm{D}}\right)$ ranging from $1.34 \pm 0.18 \times 10^{-9}$ to $1.23 \pm$ $0.27 \times 10^{-8} \mathrm{M}$ (Table 1). Compared to the immune library-derived Nbs, these three Nbs showed similarly high affinity to PCT according to SRP analysis, [23] which provided a great possibility for further antigen detection and diagnostic applications. Overall, the Nbs from the naïve library showed to have excellent affinities, completely comparable to the Nbs derived from the immune library.

\section{Assessment of Nbs binding to distinct epitopes on PCT and $\mathrm{Nb}$ biotinylation}

For the further application of the isolated $\mathrm{Nbs}$ in $\mathrm{PCT}$ detection, we built a biotin-streptavidin (SA) interactionbased ELISA. Therefore, it was necessary to firstly identify two Nbs that recognized two unique epitopes of the same antigen. By coupling the PCT Nbs with HRP and then doing the paired test, among the three Nbs, $\mathrm{Nb} 2$ and $\mathrm{Nb} 3$ were identified that recognized different epitopes. The Biotin-SA-System is one of the most widely used conjugation pairs in immunoassays, which promotes the analytical sensitivity and can profit from multiple amplification methodologies to even boost the sensitivity. To realize the application of Biotin-SASystem, we needed to modify our Nbs with biotins. In this study, we chose $\mathrm{Nb} 2$ to conjugate with biotin as the capture protein and HRP-coupled Nb3 as the chromogenic antibody used in PCT detection. In order to realize $\mathrm{Nb} 2$ biotinylation, firstly the $\mathrm{VHH}$ genes of $\mathrm{Nb} 2$ were sub-cloned into plasmid pBAD17 that contains a biotin acceptor domain (BAD). Afterwards, the recombinant plasmids were co-transformed into WK6 cells with another plasmid pBirA, which can express biotin ligase that catalyzes the ligation between biotin and BAD. BiNb2 was purified by SA Mutein Matrix and analyzed by SDS-PAGE (Figure 6A).

\section{PCT detection by a sandwich ELISA based on BiNb2}

As shown in Figure $6 \mathrm{~B}, \mathrm{BiNb} 2$ was efficiently captured by SA molecules on BeaverNano ${ }^{\text {tix }}$ SA Matrix Coated 96Well Plate as the capture antibody. PCT was recognized and captured by BiNb2, and then Nb3-HRP was used as the detector as it recognized another antigenic site. The absorbance at $450 \mathrm{~nm}$ demonstrated a high linearity in the range from 10 to $1000 \mathrm{ng} / \mathrm{mL}$ of PCT. The linear equation was calculated as $\mathrm{Y}=0.0015 \mathrm{X}+0.1597$ with an acceptable linear correlation coefficient $(r)$ of 0.9884 

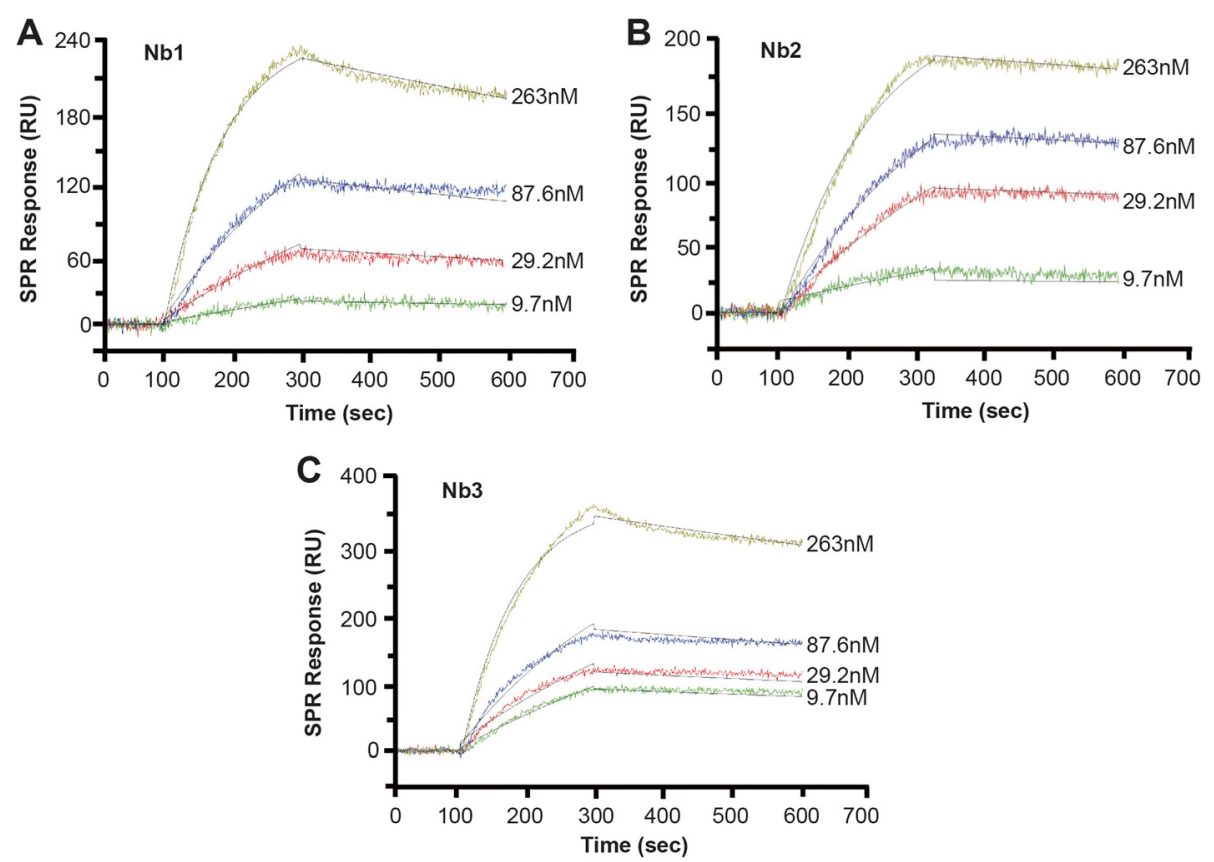

Figure 5 SPRi binding assay. Affinity between PCT and the three Nbs was determined by SPRi binding assay. (A) Nb1, (B) Nb2 and (C) Nb3 were immobilized on the chip surface and PCT in PBST were injected at the concentrations of 9.7, 29.2, 87.6 and $263 \mathrm{nM}$.

(Figure 6C). Therefore, the Nbs from the naïve library could be functional tools for the determination of disease biomarker.

\section{Real sample analysis}

In order to explore the feasibility for a real sample, the proposed ELISA was used to determine the recoveries of different concentrations $(10,100,500$ and $1000 \mathrm{ng} / \mathrm{mL})$ of PCT in serum samples by standard methods. As shown in Table 2, the recovery was in the range of $93.1-116.6 \%$ and the relative standard deviation (RSD) was in the range of $2.42-5.34 \%$. Hence, the developed sandwich ELISA has great potential for clinical application. These results indicated that the Nbs from the proposed naïve library possess practical functions and capabilities, which is crucial for the development of real applications in the future.

\section{Conclusions}

A large and functional naïve $\mathrm{VHH}$ library was constructed, from which, three Nbs families against PCT were successfully isolated. In comparison with the PCT $\mathrm{Nbs}$ from an immune library, the naïve library-derived PCT Nbs showed similar affinity and antigen-binding thermostability and thermotolerant sustainability at $37^{\circ} \mathrm{C}$ and $60^{\circ} \mathrm{C}$. Furthermore, two of the PCT Nbs were successfully used to detect PCT based on an improved sandwich ELISA based on the Biotin-SA-System. In brief, such a naïve library can be a powerful and resourceful alternative to the immune libraries. Besides, the characterized Nbs against PCT could provide great values in medical applications.

\section{Methods}

\section{Materials and instruments}

PCT was purchased from Huibiao Biological Technology Co., Ltd. Freund's adjuvant, horseradish peroxidase (HRP), goat anti-mouse IgG-alkaline phosphatase, Bis (p-nitrophenyl) phosphate (BNPP) and Tetramethylbenzidine (TMB) were purchased from Sigma-Aldrich. Mouse anti-HA tag antibody was obtained from Covance. Fast Track 2.0 Kit and ThermoScript RT-PCR Kit was obtained from InVitrogen. Pst I, Not I, Nco I, BstE II and T4 ligase were obtained from NEB (USA). Streptavidin Mutein Matrix was purchased from Roche. 96-well Maxisorp plates were purchased from Thermo Scientific NUNC. DNA markers were provided by Takara. Protein markers were obtained from Vazyme Biotech Co., Ltd and Thermo Scientific. Polyethylene glycol (PEG) 6000 and Biotin were obtained from Shanghai Sangon Biotech. BeaverNano ${ }^{\text {ma }}$ Streptavidin Matrix Coated 96-Well Plates were provided by Beaver. Phagemid vector pMECS, VCSM13 helper phages, Escherichia coli (E. coli) TG1 and WK6 cells, plasmids pBAD17 and pBirA were from Prof. Serge Muyldermans's lab (Laboratory of Cellular and Molecular Immunology, VUB-Vrije Universiteit, Brussel, Belgium). Bactrian camels were provided by "Joint Center for Nanobody Research \& 

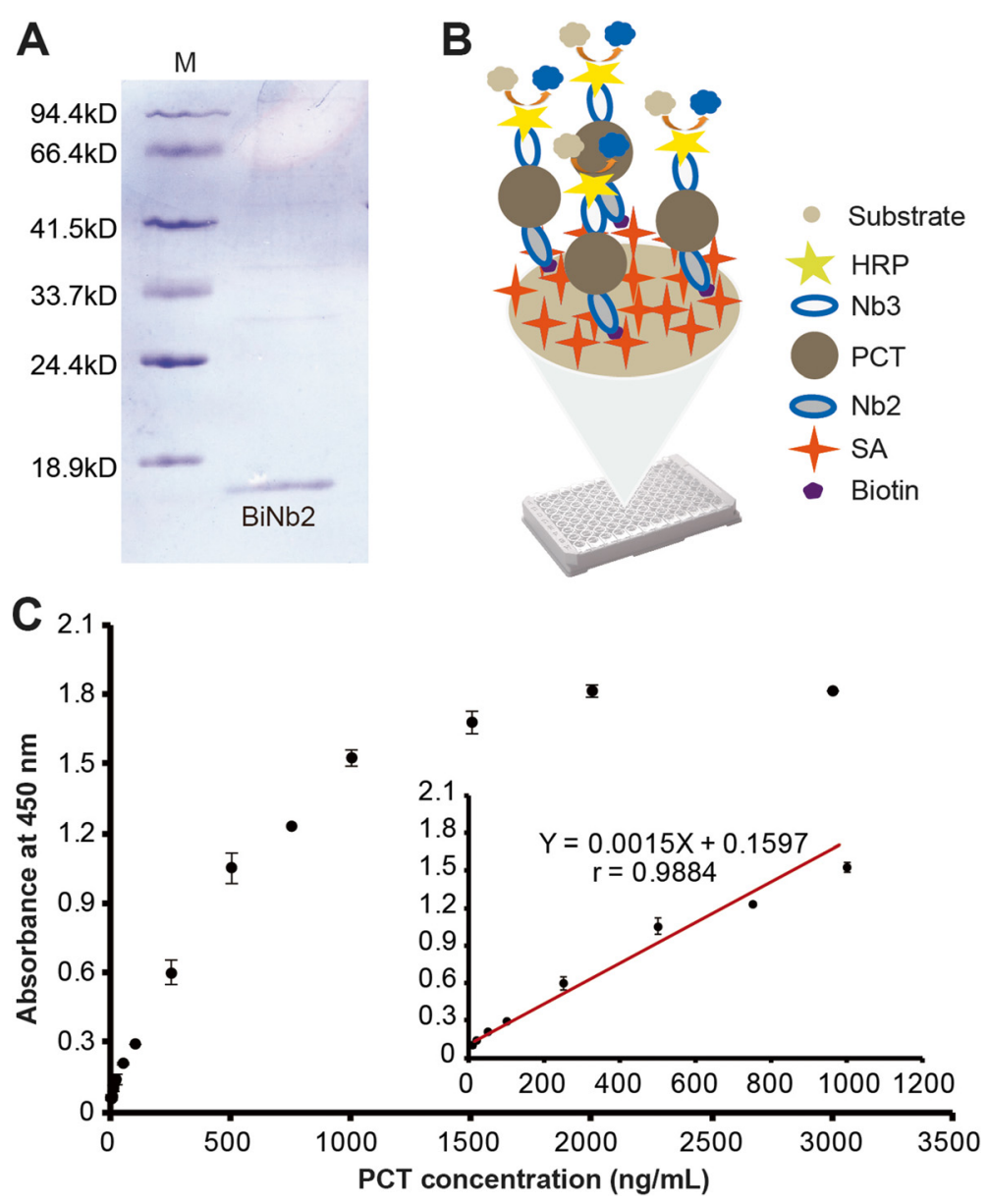

Figure 6 Detection of PCT by the sandwich ELISA based on BiNb2. (A) The purified BiNb2 was analyzed by SDS-PAGE. (B) Schematic drawing of the proposed sandwich ELISA. (C) Calibration curve toward different concentrations of PCT. The linear relationship was in the range from 10 to $1000 \mathrm{ng} / \mathrm{mL}$.

Development between SEU and Egens Bio". Affinity analysis by surface plasmon resonance imaging (SPRi) binding assay was performed on PlexArray ${ }^{\circ} \mathrm{HT}$ system (Plexera LLC).

\section{Naïve VHH library construction}

PBLs were isolated from $1 \mathrm{~L}$ of fresh blood from twenty four non-immunized bactrian camels. The camels are still alive and are kept on the farm. In the meantime, a spleen was obtained from another non-immunized

Table 2 Detection of PCT in serum samples by the proposed sandwich ELISA

\begin{tabular}{lllll}
\hline & \multicolumn{3}{l}{ PCT concentration $(\mathbf{n g} / \mathbf{m L})$} & \\
\cline { 2 - 3 } Sample & Add & Found $(\mathbf{n}=\mathbf{6})$ & Recovery (\%) & RSD (\%) $(\mathbf{n}=\mathbf{6})$ \\
\hline 1 & 10 & 11.66 & 116.6 & 5.34 \\
2 & 100 & 93.09 & 93.1 & 3.71 \\
3 & 500 & 519.09 & 103.8 & 4.86 \\
4 & 1,000 & 941.87 & 94.2 & 2.42 \\
\hline
\end{tabular}

bactrian camel. All animal experiments were carried out in accordance with the approved guidelines of Southeast University and all experiment protocols were approved by Institutional Ethic Committee of Southeast University.

Total RNA was isolated from the PBLs and the ground spleen sample using a Fast Track 2.0 Kit. The VHH library was constructed according to our previous studies $[17,25,26]$. Briefly, the cDNA was synthesized by reverse transcription (RT)-polymerase chain reaction (PCR) using a ThermoScript RT-PCR Kit with oligo- $\mathrm{dT}_{12-18}$ as the primer. Then a multi-step PCR was used to amplify VHH gene fragments. The first-step PCR was performed with the primers CALL001 and CALL002 [27]. The $600 \mathrm{bp}$ amplicons were extracted from the agarose gel and used as template. The second PCR amplfication was performed with primers VHH-Back and PMCF [28]. Afterwards, the final products were extracted from agarose gel purification. The purified products were ligated into the phagemid pMECS at $16^{\circ} \mathrm{C}$ for $16 \mathrm{~h}$ with T4 DNA ligase after digestion by Pst I and Not I. Totally, $1680 \mu \mathrm{g}$ insert DNA and 
$5600 \mu \mathrm{g}$ pMECS phagemid were used in ligations and 7000 ligations were performed. For one day's experiment, 200 ligations were performed. $48 \mu \mathrm{g}$ insert DNA and $160 \mu \mathrm{g}$ vector were used and mixed into $20 \mathrm{~mL}$ for a ligation mixture. The mixture was then divided into 200 ligations and incubated at $16^{\circ} \mathrm{C}$ for $16 \mathrm{~h}$. After ligation, we recovered the ligation products and dissolved into $1 \mathrm{~mL}$ of $\mathrm{ddH}_{2} \mathrm{O}$. The ligation products were mixed into $9 \mathrm{~mL}$ of fresh TG1 competent cells and divided into 250 electroporations to perform the transformation with the voltage of $1700 \mathrm{~V}$. In total, about 8750 electroporations were performed during the whole library construction. The cells were re-suspended into $100 \mathrm{~mL}$ of SOC medium and incubated at $37^{\circ} \mathrm{C}$ for $1 \mathrm{~h}$ after electroporations. Then the cells were re-suspended into $100 \mathrm{~mL}$ of LB medium supplemented with $15 \%(\mathrm{v} / \mathrm{v})$ glycerol and stored at $-80^{\circ} \mathrm{C}$. After all the electroporations were finished, all the cells stored at $-80^{\circ} \mathrm{C}$ were slowly thawed, mixed, centrifuged and re-suspended into $70 \mathrm{~mL}$ of LB medium. From which, $500 \mu \mathrm{L}$ of the cells was used for the identification of library size. Another $69 \mathrm{~mL}$ of the cells were diluted with LB medium and were plated onto plates containing solid LB medium supplemented with $100 \mu \mathrm{g} / \mathrm{mL}$ ampicillin and $2 \%(\mathrm{w} / \mathrm{v})$ glucose, and cultured at $37^{\circ} \mathrm{C}$ overnight. Finally, the colonies were scraped into $300 \mathrm{~mL}$ of liquid LB medium supplemented with $15 \%$ (v/v) glycerol and were stored at $-80^{\circ} \mathrm{C}$.

\section{Selection of PCT Nbs}

Based on the successful construction of the naïve library, we used PCT as the target protein to isolate the antigen binders. We only took a smaller sample of the library to investigate whether good quality of binders can be isolated. It is obvious that small percent of library may lose the diversity of Nbs during selection, which was also reported in a previous study [24]. About $1 \times 10^{10} \mathrm{TG}$ cells from the library stock were grown in $2 \times \mathrm{TY}$ medium containing $100 \mu \mathrm{g} / \mathrm{mL}$ ampicillin and $1 \%(\mathrm{w} / \mathrm{v})$ glucose at $37^{\circ} \mathrm{C}$. After $2 \mathrm{~h}$, the cells were infected with $1 \times 10^{12}$ VCSM13 helper phages for $30 \mathrm{~min}$ at room temperature (RT). The infected cells were harvested and resuspended into $2 \times \mathrm{TY}$ medium supplemented with $100 \mu \mathrm{g} / \mathrm{mL}$ ampicillin and $70 \mu \mathrm{g} / \mathrm{mL}$ kanamycin, then the cells were incubated overnight at $37^{\circ} \mathrm{C}$ and $220 \mathrm{rpm}$. The phages were precipitated from culture supernatant with PEG $6000 / \mathrm{NaCl}$ and resuspended in sterile PBS. About $2 \times$ $10^{11}$ phage particles were used for each bio-panning against PCT $(200 \mu \mathrm{g} / \mathrm{mL})$ coated on 96-well Maxisorp plates. After four consecutive rounds of bio-panning, 95 individual colonies were randomly chosen and each was grown in $1 \mathrm{~mL}$ of terrific broth (TB) medium. The expression of $\mathrm{Nbs}$ was induced with $1 \mathrm{mM}$ isopropyl $\beta$-D-1-thiogalactopyranoside (IPTG) overnight at $28^{\circ} \mathrm{C}$. Positive clones expressing anti-PCT Nbs were identified by PE-ELISA. Then the identified clones were sent for sequencing. The amino acids sequences of $\mathrm{Nbs}$ genes were analyzed and classified into different families based on their sequence diversity in CDR3 regions.

\section{Expression and purification of PCT Nbs}

The plasmids of the different $\mathrm{Nb}$ families were extracted from TG1 cells and electro-transformed into E. coli WK6 cells. The WK6 cells were cultured in $330 \mathrm{~mL}$ of TB medium supplemented with $0.1 \%(\mathrm{w} / \mathrm{v})$ glucose, $100 \mu \mathrm{g} / \mathrm{mL}$ ampicillin and $2 \mathrm{mM} \mathrm{MgCl}$ till an $\mathrm{OD}_{600}$ of 0.6 , and induced with $1 \mathrm{mM}$ IPTG overnight at $28^{\circ} \mathrm{C}$. The periplasmic proteins were released by osmotic shock from the cells. The PCT-specific Nbs were produced as Cterminal hexahistidine $\left(\mathrm{His}_{6}\right)$-tag proteins and purified with $500 \mathrm{mM}$ imidazole solution. Afterwards, the Nbs were ultra-filtrated to remove the imidazole molecules and dialyzed into PBS solution. Ultimately, the purified PCT-specific Nbs were diluted to $1 \mathrm{mg} / \mathrm{mL}$ as the stock and analyzed by sodium dodecyl sulfate polyacrylamide gel electrophoresis (SDS-PAGE).

\section{PCT-binding thermostability analysis}

Heat resistance of the naïve library-derived PCT Nbs and the immune library-derived PCT Nbs were assessed by incubating them at $37^{\circ} \mathrm{C}$ for various times $(0,12,24$, 48,60 and $72 \mathrm{~h}$ ), at $60^{\circ} \mathrm{C}$ for $0.5,1,2,3,4,5$ and $6 \mathrm{~h}$, and at $90^{\circ} \mathrm{C}$ for $20,40,60,90,120,150$ and $180 \mathrm{~min}$. 96-well Maxisorp plates were coated with $100 \mu \mathrm{L}$ of $5 \mu \mathrm{g} / \mathrm{mL}$ PCT in coating buffer $(0.1 \mathrm{M} \mathrm{NaHCO}$, $\mathrm{pH}$ 9.6), and the corresponding blank controls were set. After blocking with $1 \%(\mathrm{w} / \mathrm{v})$ skim milk for $2 \mathrm{~h}, 100 \mu \mathrm{L}$ of Nbs solution $(10 \mu \mathrm{g} / \mathrm{mL})$ were added into the wells to incubate for $1 \mathrm{~h}$. After washing, mouse anti-HA tag antibody was added for $1 \mathrm{~h}$ followed by goat anti-mouse IgG-alkaline phosphatase for $1 \mathrm{~h}$ at RT. After 5 times of washing with PBST (PBS with $0.05 \%$ tween-20), BNPP substrate solution was added and finally the plates were read using Bio-Rad iMark ${ }^{\mathrm{TM}}$ (Bio-Rad, USA) at $405 \mathrm{~nm}$.

\section{Affinity analysis by SPRi binding assay}

SPRi binding assay was performed according to our previous study [17]. Briefly, the Nbs were spotted on the NanoCapture 3D-chip surface for $1 \mathrm{~h}$ to immobilize. Afterwards, the chip was incubated in $2 \mathrm{~mL}$ of $1 \mathrm{M}$ ethanolamine- $\mathrm{HCl}(\mathrm{pH}$ 8.5) for $20 \mathrm{~min}$ in order to absolutely block the remaining activated NHS groups. The chip was loaded into PlexArray ${ }^{\circ} \mathrm{HT}$, and flew PBST as running buffer at a constant flow rate for setting up assay. 4 series of PCT dilutions (9.7, 29.2, 87.6 and 263.0 $\mathrm{nM})$ were sequentially injected at a flow rate of $2 \mu \mathrm{L} / \mathrm{s}$. Finally, in order to elute the bound analytes for returning to baseline value, the chip was then regenerated by 1:200 (v/v) $\mathrm{H}_{3} \mathrm{PO}_{4}$ at a flow rate of $3 \mu \mathrm{L} / \mathrm{s}$. The whole 
assays were conducted at the same conditions as during the set up. All binding data were analyzed using Plexera Data Analysis Module. Binding curves were fitted with 1:1 Langmuir binding model for a binding kinetics.

\section{HRP coupling}

In order to determine whether the isolated PCT Nbs could recognize different epitopes of PCT, HRP was used to couple to the purified Nbs firstly as described in our previous study [25]. Briefly, $100 \mu \mathrm{L}$ of fresh NaIO4 $(0.1 \mathrm{M})$ was incubated with $200 \mu \mathrm{L}$ of $5 \mathrm{mg} / \mathrm{mL}$ HRP solution for $30 \mathrm{~min}$ at $4^{\circ} \mathrm{C}$. Next, $100 \mu \mathrm{L}$ of $2.5 \%(\mathrm{v} / \mathrm{v})$ ethylene glycol were added and incubated for $30 \mathrm{~min}$ at RT. Then $1 \mathrm{~mL}$ of PCT Nbs $(1 \mathrm{mg} / \mathrm{mL})$ was added and the mixture was incubated at $4^{\circ} \mathrm{C}$ overnight in the dark. Afterwards, $20 \mu \mathrm{L}$ of $\mathrm{NaBH}_{4}(5 \mathrm{mg} / \mathrm{mL})$ were mixed into the $\mathrm{PCT} / \mathrm{HRP}$ mixture for $3 \mathrm{~h}$ at $4^{\circ} \mathrm{C}$. Finally the mixture was transferred to an Ultra-filtration column to replace the buffer with PBS.

\section{Assessment of Nbs binding to distinct epitopes on PCT}

In order to identify two Nbs that can recognize distinct epitopes on PCT, an indirect ELISA was performed. A 96-well Maxisorp plate was coated with $5 \mu \mathrm{g} / \mathrm{mL} \mathrm{Nbs}$ in $0.1 \mathrm{M} \mathrm{NaHCO}_{3}(\mathrm{pH} 9.6)$ at $4^{\circ} \mathrm{C}$ overnight. After washing with PBST and blocking with $3 \%(\mathrm{w} / \mathrm{v})$ bovine serum albumin (BSA), $100 \mu \mathrm{L}$ of PCT $(2 \mu \mathrm{g} / \mathrm{mL})$ was added to the wells and PBS was added to the corresponding wells as the control for $1 \mathrm{~h}$. The HRP-conjugated Nbs $(2 \mu \mathrm{g} / \mathrm{mL})$ were added to the wells. After several times of washing by PBST, $100 \mu \mathrm{L}$ of TMB solution was added and incubated for 5-10 $\mathrm{min}$ at $\mathrm{RT}$. The enzyme reaction was stopped by $50 \mu \mathrm{L}$ of $2 \mathrm{M} \mathrm{H}_{2} \mathrm{SO}_{4}$ and the absorbance was read at $450 \mathrm{~nm}$.

\section{$\mathrm{Nb}$ biotinylation in vivo}

According to the results of epitope mapping, we chose PCT Nb2 to conjugate with biotin. The biotinylation was performed as our previous studies described [17,25]. Briefly, the gene encoding PCT Nb2 was sub-cloned into plasmid pBAD17 and the recombinant plasmid was coelectrotransformed into competent WK6 cells with the plasmid pBirA. The cells were cultured in $330 \mathrm{~mL}$ of TB medium and $330 \mu \mathrm{L}$ of $50 \mathrm{mM}$ biotin was added when the OD was increased to about 0.4. The expression of proteins was induced by $1 \mathrm{mM}$ IPTG overnight at $28^{\circ} \mathrm{C}$. The biotinylated PCT Nb2 (BiNb2) was purified by SA Mutein Matrix with elution buffer containing $4 \mathrm{mM}$ biotin. The residual biotin molecules were removed by Ultra-filtration column with PBS for several times.

\section{PCT detection by a sandwich ELISA based on BiNb2} $100 \mu \mathrm{L}$ of BiNb2 $(2 \mu \mathrm{g} / \mathrm{mL})$ in PBST was added per well of a BeaverNano ${ }^{\mathrm{Tm}}$ SA Matrix Coated 96-Well Plate at
RT for $1.5 \mathrm{~h}$. After 5 times of washing with PBST and blocking with 5\% BSA in PBST for $1 \mathrm{~h}$, the wells were incubated with serial dilutions $(0,1,2,5,10,20,50,100$, 250, 500, 750, 1000, 1500, 2000 and $3000 \mathrm{ng} / \mathrm{mL}$ ) of PCT for $1 \mathrm{~h}$. Afterwards, $100 \mu \mathrm{L}$ of PCT Nb3 conjugated to HRP (Nb3-HRP) $(1 \mu \mathrm{g} / \mathrm{mL})$ in PBST containing $5 \%$ BSA was added. After $1 \mathrm{~h}, 200 \mu \mathrm{L}$ of PBST was added to the wells, and the plate was shaken for $5 \mathrm{~min}$ at RT. 10 times of washing were repeated and $100 \mu \mathrm{L}$ of TMB solution was added to incubate for $10 \mathrm{~min}$ at RT. Finally, the absorbance was read at $450 \mathrm{~nm}$ after the enzyme reaction was stopped by addition of $50 \mu \mathrm{L}$ of $2 \mathrm{M} \mathrm{H}_{2} \mathrm{SO}_{4}$ solution.

\section{Additional file}

Additional file 1: Supplemental data for this article can be accessed on the publisher's website.

\section{Abbreviations}

Nb: Nanobody; Nbs: Nanobodies; VHH: Variable domain of the heavy chain of the heavy-chain only antibody; PCT: Procalcitonin; IgG: Immunoglobulin G; SDS-PAGE: Sodium dodecyl sulfate polyacrylamide gel electrophoresis; Fabs: antigen-binding fragments; scFvs: Single-chain antibody fragments; HCAbs: Heavy chain-only antibodies; PBLs: Peripheral blood lymphocytes; CFU: Colony-forming units; CDR: Complementarity determining region; BSA: Bovine serum albumin; E. coli: Escherichia coli; HRP: Horseradish peroxidase; ELISA: Enzyme-linked immunosorbent assay; SRPi: Surface plasmon resonance imaging; IPTG: Isopropyl- $\beta$-d-thiogalactoside; His.: hexahistidine; HA: Hemagglutinin; $\mathrm{pH}$ : Potential of hydrogen; PEELISA: Periplasmic extract ELISA; BNPP: Bis (p-nitrophenyl) phosphate; TMB: 3, 3', 5, 5'-Tetramethylbenzidine; SA: Streptavidin; TB: Terrific broth.

\section{Competing interests}

The authors declare that they have no competing interests.

\section{Authors' contributions}

$J Y$ performed the experiments and wrote the manuscript. PW and MZ helped with the experiments and parts of the manuscript writing. GL helped with data analysis and figures preparation. ER helped with manuscript writing and language revision. SX and YW supervised the experiments, reviewed the manuscript and provided the funding. All authors read and approved the final manuscript.

\section{Acknowledgements}

This work was supported by Jiangsu Nanobody Engineering and Research Center of China (Grant 2015-04), Program for New Century Excellent Talents in University (NCET-20130127), National Natural Science Foundation of China (Grant 31271365 and 31471216) to Yakun Wan. National Project for Significant New Drugs Development of the MOST of China (2012ZX09103- 301-033 and 2012ZX09202-301-001) and the Major Biotech Industrialization Projects from the Guangzhou Municipal Science and Technology Bureau (2010U1-E00541) to Xiong Sheng. We would like to thank Hong Liu from Plexera LLC (Beijing) for providing support on SPRi binding assay.

\section{Author details}

The Key Laboratory of Developmental Genes and Human Disease, Ministry of Education, Institute of Life Sciences, Southeast University, Nanjing 210096, PR China. ${ }^{2}$ Jiangsu Nanobody Engineering and Research Center, Nantong 226010, PR China. ${ }^{3}$ Institute of Biomedicine \& National Engineering Research Center of Genetic Medicine, College of Life Science and Technology, Jinan University, Guangzhou 510630, PR China. ${ }^{4}$ Laboratory of Cellular and Molecular Immunology, Vrije Universiteit Brussel, Faculty of Science, Pleinlaan 2, 1050, Brussels, Belgium. 
Received: 13 January 2015 Accepted: 15 April 2015

Published online: 06 May 2015

\section{References}

1. Fernandez LA, Muyldermans S. Recent developments in engineering and delivery of protein and antibody therapeutics. Curr Opin Biotechnol. 2011;22:839-42

2. Saerens D, Frederix F, Reekmans G, Conrath K, Jans K, Brys L, et al. Engineering camel single-domain antibodies and immobilization chemistry for human prostate-specific antigen sensing. Anal Chem. 2005;77:7547-55.

3. Market E, Papavasiliou FN. V(D)J recombination and the evolution of the adaptive immune system. PLoS Biol. 2003;1, E16.

4. Hassanzadeh-Ghassabeh G, Devoogdt N, De Pauw P, Vincke C, Muyldermans S. Nanobodies and their potential applications. Nanomedicine (Lond). 2013;8:1013-26.

5. Holt LJ, Herring C, Jespers LS, Woolven BP, Tomlinson IM. Domain antibodies: proteins for therapy. Trends Biotechnol. 2003;21:484-90.

6. Nelson AL. Antibody fragments: hope and hype. MAbs. 2010;2:77-83.

7. Zhang J, Tanha J, Hirama T, Khieu NH, To R, Tong-Sevinc H, et al. Pentamerization of single-domain antibodies from phage libraries: a novel strategy for the rapid generation of high-avidity antibody reagents. J Mol Biol. 2004:335:49-56.

8. Xia J, Zhang Y, Qian J, Zhu X, Zhang Y, Zhang J, et al. Isolation, identification and expression of specific human CD133 antibodies. Sci Rep. 2013:3:3320

9. Doshi $R$, Chen BR, Vibat CR, Huang N, Lee CW, Chang G. In vitro nanobody discovery for integral membrane protein targets. Sci Rep. 2014;4:6760.

10. Ebrahimizadeh W, Mousavi Gargari S, Rajabibazl M, Safaee Ardekani L, Zare $\mathrm{H}$, Bakherad H. Isolation and characterization of protective anti-LPS nanobody against $\mathrm{V}$. cholerae $\mathrm{O} 1$ recognizing Inaba and Ogawa serotypes. Appl Microbiol Biotechnol. 2013;97:4457-66.

11. Hamers-Casterman C, Atarhouch T, Muyldermans S, Robinson G, Hamers C, Songa EB, et al. Naturally occurring antibodies devoid of light chains. Nature. 1993;363:446-8.

12. Greenberg AS, Avila D, Hughes M, Hughes A, McKinney EC, Flajnik MF. A new antigen receptor gene family that undergoes rearrangement and extensive somatic diversification in sharks. Nature. 1995;374:168-73.

13. Van de Broek B, Devoogdt N, D'Hollander A, Gijs HL, Jans K, Lagae L, et al. Specific cell targeting with nanobody conjugated branched gold nanoparticles for photothermal therapy. ACS Nano. 2011;5:4319-28.

14. van der Linden RH, Frenken LG, de Geus B, Harmsen MM, Ruuls RC, Stok W, et al. Comparison of physical chemical properties of llama VHH antibody fragments and mouse monoclonal antibodies. Biochim Biophys Acta. 1999;1431:37-46.

15. Stewart CS, MacKenzie CR, Hall JC. Isolation, characterization and pentamerization of alpha-cobrotoxin specific single-domain antibodies from a naive phage display library: preliminary findings for antivenom development. Toxicon. 2007:49:699-709.

16. Goldman ER, Anderson GP, Liu JL, Delehanty JB, Sherwood LJ, Osborn LE, et al. Facile generation of heat-stable antiviral and antitoxin single domain antibodies from a semisynthetic llama library. Anal Chem. 2006;78:8245-55.

17. Li H, Yan J, Ou W, Liu H, Liu S, Wan Y. Construction of a biotinylated cameloid-like antibody for lable-free detection of apolipoprotein B-100. Biosens Bioelectron. 2014;64C:111-8.

18. Verheesen $P$, ten Haaft MR, Lindner $N$, Verrips $C T$, de Haard JJ. Beneficial properties of single-domain antibody fragments for application in immunoaffinity purification and immuno-perfusion chromatography. Biochim Biophys Acta. 2003;1624:21-8.

19. Yardehnavi N, Behdani M, Pooshang Bagheri K, Mahmoodzadeh A, Khanahmad H, Shahbazzadeh D, Habibi-Anbouhi M, Ghassabeh GH, Muyldermans S: A camelid antibody candidate for development of a therapeutic agent against Hemiscorpius lepturus envenomation. FASEB 2014 28:4004-14

20. Monegal A, Ami D, Martinelli C, Huang H, Aliprandi M, Capasso P, et al. Immunological applications of single-domain llama recombinant antibodies isolated from a naive library. Protein Eng Des Sel. 2009;22:273-80.

21. Osthoff M, Eisen DP. Procalcitonin as a diagnostic marker for sepsis. Lancet Infect Dis. 2013;13:1013-4.

22. Christ-Crain M, Jaccard-Stolz D, Bingisser R, Gencay MM, Huber PR, Tamm M, et al. Effect of procalcitonin-guided treatment on antibiotic use and outcome in lower respiratory tract infections: cluster-randomised, single-blinded intervention trial. Lancet. 2004;363:600-7.

23. Li H, Sun Y, Elseviers J, Muyldermans S, Liu S, Wan Y. A nanobody-based electrochemiluminescent immunosensor for sensitive detection of human procalcitonin. Analyst. 2014;139:3718-21.

24. Griffiths AD, Williams SC, Hartley O, Tomlinson IM, Waterhouse P, Crosby WL, et al. Isolation of high affinity human antibodies directly from large synthetic repertoires. EMBO J. 1994;13:3245-60.

25. Zhu M, Hu Y, Li G, Ou W, Mao P, Xin S, et al. Combining magnetic nanoparticle with biotinylated nanobodies for rapid and sensitive detection of influenza H3N2. Nanoscale Res Lett. 2014:9:528.

26. Ma L, Sun Y, Kang X, Wan Y. Development of nanobody-based flow injection chemiluminescence immunoassay for sensitive detection of human prealbumin. Biosens Bioelectron. 2014;61:165-71.

27. Conrath KE, Lauwereys M, Galleni M, Matagne A, Frere JM, Kinne J, et al. Beta-lactamase inhibitors derived from single-domain antibody fragments elicited in the camelidae. Antimicrob Agents Chemother. 2001:45:2807-12

28. Vincke C, Gutierrez C, Wernery U, Devoogdt N, Hassanzadeh-Ghassabeh G, Muyldermans S. Generation of single domain antibody fragments derived from camelids and generation of manifold constructs. Methods Mol Biol. 2012;907:145-76.

\section{Submit your next manuscript to BioMed Central and take full advantage of:}

- Convenient online submission

- Thorough peer review

- No space constraints or color figure charges

- Immediate publication on acceptance

- Inclusion in PubMed, CAS, Scopus and Google Scholar

- Research which is freely available for redistribution 